\title{
ITS and trnL-F Sequences analysis of Potentilla discolor Bge.
}

\author{
Shaoxuan Zhang ${ }^{1, ~ a ~, ~ " Y a r o n g ~ L i ~}{ }^{2, b}$, Tiantian Wang ${ }^{1, c}$, Guangzhu Lin ${ }^{3, d}$, \\ Bochuan Wang ${ }^{1, e}$ \\ ${ }^{1}$ Laboratory of Molecular Genetics, Institute of Frontier Medical Sciences, Jilin University, Xinmin \\ Street 1163\#, Changchun Jilin China 130021 \\ ${ }^{2}$ Department of Oncology and Hematology, The Second Bethune Hospital, Jilin University, Ziqiang \\ Street 218\#, Changchun Jilin China 130041 \\ ${ }^{3}$ Cardiolvascular Disease Diagnosis and Treatment Center, The First Bethune Hospital, Jilin \\ University, Xinmin Street 71\#, Changchun Jilin China 130021 \\ azhangshaoxuan@jlu.edu.cn bdoctor.lyr@163.com cttw14@mail.jlu.edu.cn ${ }^{d}$ lingz6403@163.com \\ ewangbc11@163.com \\ Corresponding Author
}

Keywords: Potentilla discolor Bge.; ITS; trnL-F; Sequencing: Authentication.

Abstract. To provide scientific data of the internal transcribed spacer (ITS) and trnL-F sequences for the authentication of Potentilla discolor Bge., we extracted the genome DNA from the leaves of Potentilla discolor Bge., purchased from Hebei Province, amplified the ITS region using ITS universal primers of angiosperm, and sequenced the purified PCR products directly. The obtained sequences were edited by Genetyx and reported here.

\section{Introduction}

Discolor Cinquefoil Herb, the dried herb of Potentilla discolor Bge.(Rosaceae), has the functions of heat-clearing and detoxifying; arresting bleeding; curing dysentery and counteracting toxicity. It has been introduced widely, especially in the scientific book 《Chinese Materia Medica》 ${ }^{[1]}$. Recently, it is found to have function of curing diabetes. Due to these reasons it started to be prescribed from 2010 edition of 《Pharmacopoeia of The People's Republic of China $\rangle^{[2]}$. Due to the similar morphology and other reasons, it is often mis-collected and used as Chinese Cinquefoil(Potentilla chinesis Ser.) ${ }^{[3]}$ and even Chinese Anemone(Pulsatilla chinesis(Bge) Regel. $)^{[4]}$. These lead to mistakes in application and affect the therapeutic effect of Discolor Cinquefoil Herb. So authentication of Potentilla discolor Bge. from the others is necessary for correct use of this drug.Although methods based on traditional appearance, microscopy and thin-layer chromatography have been established and even improved, but due to the similar morphology and the same chemical composition, it is very hard to authenticate Discolor Cinquefoil Herb from the others used as it by them. Recently, as a result of progresses in molecular biology, molecular authentication has become a new and very relaible method. An accurate method of molecular authentication is generally established based on the data of DNA sequences, such as ITS and chloroplast genes like trnL-F, so sequencing of these regions of Potentilla discolor Bge. and the other plants that are used as it becomes the most foundamental step. In this study, we sequenced the ITS and trnL-F region of Potentilla discolor Bge.. The obtained sequences were edited and reported here.

\section{Materials and Methods}

\section{Materials}

Potentilla discolor Bge. was purchased from YAODUBAICAOYANGSHENGTANG, Baoding, Hebei, China. The sample was authenticated by Professor Wenchang Guo of Jilin University (Table 1). The leaves dried by silica gel were used for DNA extraction. 


\section{DNA Extraction}

$0.3 \mathrm{~g}$ leaf was taken from every sample, and put into a culture dish. The leaves were cleaned with cotton swabs to wipe out the impurities with $70 \%$ of alcohol. The dried leaves were then grinded to powders in liquid nitrogen and used to extract genome DNA using Plant DNA Isolation Reagent (Takara Biotechnology (Dalian) Co., Ltd., Dalian, China) following the manufacturer's manual. The extracted genome DNA was dissolved in adequate volume of ultrapure water. The quality of the DNA was checked by $1 \%$ agarose gel electrophoresis with $5 \mu \mathrm{L}$ of each solution.

\section{Primer Design}

The universal primers of ITS ${ }^{[5]}$ and trnL-F ${ }^{[6]}$ were selected and synthesized by Takara Biotechnology (Dalian) Co., Ltd., (Dalian China). The sequences are as follows:

PCR primesrs:

For ITS:

ITS-F: 5'-TCC ACT GAA CCT TAT CAT TTA G-3'

ITS-R: 5'-CCA TGC TTA AAC TCA GCG GGT-3'

For trnL-F:

trnLF-cF: 5'-CGAAATCGGTAGACGCTACG-3'

trnLK-fR: 5'-ATI'TGAACTGGTGACACGAG-3'

Sequencing primers:

For ITS:

ITS-F: 5'-TCC ACT GAA CCT TAT CAT TTA G-3'

In-ITS-3'R: 5'-GAC TCG ATG GTT CAC GGG ATT CT-3'

In-ITS-5'F: 5'-TCT CGC ATC GAT GAA GAA CG-3'

ITS-R: 5'-CCA TGC TTA AAC TCA GCG GGT-3'

For trnL-F:

trnLF-cF: 5'-CGAAATCGGTAGACGCTACG-3'

trnLF-dR: 5'-GGGGATAGAGGGACTTGAAC-3'

trnLK-eF: 5'-GGTTCAAGTCCCTCTATCCC-3'

trnLK-fR: 5'-ATI'TGAACTGGTGACACGAG-3'

\section{PCR Amplification}

PCR was performed in a $50 \mu \mathrm{L}$ reaction mixture containing $1 \mu \mathrm{L}$ of each 2.3 PCR primer, $5 \mu \mathrm{L}$ of template DNA, $5 \mu \mathrm{L}$ of $10 \times$ Buffer, $5 \mu \mathrm{L}$ of dNTPs, $1 \mu \mathrm{L}$ of Taq DNA polymerase (Takara Biotechnology (Dalian) Co., Ltd., Dalian China). PCR was performed in the following conditions: 1 cylce of $94^{\circ} \mathrm{C}$ for $5 \mathrm{~min}, 35$ cycles of denature at $94^{\circ} \mathrm{C}$ for $1 \mathrm{~min}$, annealing at $55^{\circ} \mathrm{C}$ for $2 \mathrm{~min}$, extension at $72^{\circ} \mathrm{C}$ for $2 \mathrm{~min}$, 1 cycle of $72^{\circ} \mathrm{C}$ for $10 \mathrm{~min}$. All the reactions were performed using MiniCycler PTC-150(MJ Research Inc, St. Bruno, Canada). All the PCR products were checked using a 1\% agarose gel.

\section{Sequencing}

2.4 PCR products were purified using Montage PCR Filter Units (Millipore Corporation, USA.) and then sequenced. Sequencing reaction was performed in a $10 \mu \mathrm{L}$ reaction mixture by using ABI BigDye Terminator v3.1 Cycle Sequencing Kit(Applied Biosystems, USA.) with each of the sequencing primers. Sequencing reaction was performed in the following conditions: 1 cycle of $96^{\circ} \mathrm{C}$ for $1 \mathrm{~min}, 25$ cycles of denature at $96^{\circ} \mathrm{C}$ for $30 \mathrm{sec}$, extension $50^{\circ} \mathrm{C}$ for $5 \mathrm{sec} .1$ cycle of $60^{\circ} \mathrm{C}$ for $4 \mathrm{~min}$. After purification, aequences were analyzed by using 3130 sequencer (Applied Biosystems, USA.).

\section{Sequence Analysis}

Genetyx-SV/RC version 11.0 (Software Development Co., Ltd., Tokyo, Japan) were used for editing of the sequences. 


\section{RESULTS}

The information of the purchased plants is shown in Table 1. Three individuals of each sample were used for sequence analysis.

Table 1. the location and date of sample collection

\begin{tabular}{ccc}
\hline Species & \multicolumn{1}{c}{ Source } & $\begin{array}{c}\text { Date of } \\
\text { Purchase }\end{array}$ \\
\hline $\begin{array}{c}\text { Potentilla } \\
\text { discolor }\end{array}$ & YAODUBAI & CAO \\
Bge. & YANGSHENGT & \\
\multicolumn{3}{c}{ ANG } \\
\hline
\end{tabular}

The ITS sequences of Potentilla discolor Bge. are as follows:

TCGAAACCTGCCTAGCAGAACGACCCGAGAACGTGTTTCAACGCTTGGRGACGGGGGG CCTCGCGGCTCCTCGCCTCCTTATCCCGGGAAGGGAAGCCTCGCGCGTCGTGCTTCGGC GCTTCCGCTTGGCTGACCTCTCCGGGCGTACTGAACATCGGCGTGAATTGCGCCAAGGA ACTTGAATGAAAGAGCGTCCCCCCGCCGTCTCCGGAGACGGAGACCGCGCGGGTGGTTC GTCGTCTTCAATATGTCTAAACGACTCTCGGCAACGGATATCTCGGCTCTCGCATCGATGA AGAACGTAGCGAAATGCGATACTTGGTGTGAATTGCAGAATCCCGTGAACCATCGAGTTT TTGAACGCAAGTTGCGCCCGAAGCCACTAGGCCGAGGGCACGTCTGCCTGGGCGTCACA CGTCGTTGCCCCTCCCAACCCCTCCGGGAGTTGGCTGGGACGGATGATGGCCTCCCGTG CGCTCCGTCGCGCGGTTGGCATAAATAACAAKTCCTCGGCGGCCAACGCCGCGACAATC GGTGGTTGTCAAACCTCGGTGTCCTGTCGCGTGCGAGTCGTCTGGGGCTTTTCCAATCTG ATGCGCGTCGATTCGTCGGCGCTTTCAAC

The trnL-F sequences of Potentilla discolor Bge. are as follows:

GCTACGGACTTAATTGGATTGAGCCTTGGTATGGAAACCTACCAAGTGATAACTTTCAAAT TCAGAGAAACCCCGGAATTAAAAATGGGCAATCCTGAGCCAAATCCCGTTTTATGAAAAC AAACAAGGGTTTCATAAAGCGAGAATAAATAAAGGATAGGTGCAGAGACTCAATGGAAG CTGTTYTAACAAATGGAGTTGGCTGCATTGTGTTCATAAAGGAATCCTTCCATCGAAACTT CCGAAAAGATGAAAGATAAACCTATATACATATGTATACTTACGGATGTATACTTACGGAAA TACTATCGCCAAATGATTAAAAATGATTAATGACGACTCCAACCGGTTCTATAATTTTTTTC TATCTATTTATATGATAGAAAAAAAAAGAATTAAATATTCATTGATCAAAACATTCACTCCA TCATAGTCCGATAAATCTTTTTATTTTGAAGAATTTTTTAATCGGATTAAGAATAAAGATAG AGTCCCATTTTACATGTCAATATCGACAACAAAGAAATTTATAGTAAGAGGAAAATCCGTC GATTTTAGAAATCGTGAGGGTTCAAGTCCCTCTATCCCCAAAAAGACCTGGTTGCCTCCC TAATTATTTATCTTCTCATTTTGTTAGTGACTCAAAATTGTTTATAGTTCTTAGTCATTCTCA CTCTACTATTTCATAAACCGATCTGAGCGGAAATTTTTTTTATTATCACATCATAAGCCTTAT ATGTGATATATATGATACGTGTACAAATGAGCATCTTTGAATAATGTAATAAAATTAAATAAT TAACAATCCATATCATTATTTGTATTATTTGTACTGTATTGAAACTTACAAAGTTTTCTTTTT GAAAATACAAGAAATTCTACCAGGGCCTGGATATTACTTTGTAATATCTTTTCATTTTTTTA ATTGACATAGACCCAAGTCCTATATTAAAATAAAATGAGGATGATGCGTCGTGAATGGTCG GGATAGCTCAGCTGGTAGAGCA 


\section{CONCLUSION}

The ITS and trnL-F sequences of Potentilla discolor Bge. collected in China is for the first time reported.

This study provided scientific data for molecular authentication of Potentilla discolor Bge.

Potentilla discolor Bge. distributed in wide area of China, there should be differences among the samples collected in different area, so this study is just a beginning. For authenticating Potentilla discolor Bge. more precisely, enlargement of sample from various area and accumulation of these data should be necessary in the future.

\section{REFERENCES}

[1] Guojun Xu, Luoshan Xu, Hongxian He et al. Chinese Materia Medica. Herba Potentillae discoloris: 1436-1438. Beijing: People's Medical Publishing House. (in Chinese).

[2] Chinese Pharmacopoeia Commisson. 2010. Pharmacopoeia of The People's Republic of China. In Kuang L.J. \& Zhang K.(eds.), Potentillae discolor Herba: 359-360. Beijing: People's Medical Publishing House. (in Chinese).

[3] Wan, H., Hu, Y.E.2007. Identification between Potentillae discoloris and Chinese Cinquefoil. Guiding Journal of TCM. 13(9):85 (in Chinese).

[4] Zhang, D.Y., Fu, X., Yang, Y.Y.2000. Textual research on Potentillae discoloris and it's chaos varieties. Chinese Traditional and Herbal Drugs. 31(7):554-556. (in Chinese)

[5] Takaiwa, F., Oono, K., Sugiura, M.1985.Nucleotide sequence of the 17S-25S spacer region from rice rDNA. Plant Molecular Biology. 4:355-364.

[6] Taberlet,P., Gielly,L., Pautou, G., et al . 1991. Universal Primers for Amplification of Three Non-Coding Regions of Chloroplast DNA. Plant Molecular Biology. 17:1105-1109. 